\title{
Implementation of Optimal Thermal Radiation Pumps Using Adiabatically Modulated Photonic Cavities
}

\author{
Lucas J. Fernández-Alcázar,* Huanan Li, Mona Nafari, and Tsampikos Kottos
}

Cite This: ACS Photonics 2021, 8, 2973-2979

Read Online

ABSTRACT: We numerically implement the concept of thermal radiation pumps in realistic photonic circuits and demonstrate their efficiency to control the radiation current, emitted between two reservoirs with equal temperature. The proposed pumping scheme involves a cyclic adiabatic modulation of two parameters that control the spectral characteristics of the photonic circuit. We show that the resulting pumping cycle exhibits maximum radiation current when a cyclic modulation of the system is properly engineered to be in the proximity of a resonance degeneracy in the
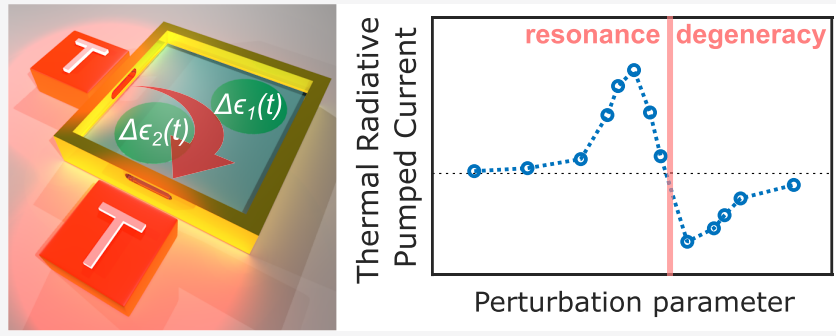
parameter space of the photonic circuit. A developed Floquet scattering framework, which in the adiabatic limit boils down to the analysis of an instantaneous scattering matrix, is offering an engineering tool for designing and predicting the performance of such thermal pumps. Our predictions are confirmed by timedomain simulations invoking an adiabatically driven photonic cavity.

KEYWORDS: thermal radiation, adiabatic pumping, driven systems, Floquet scattering, thermal photonics

$\mathrm{T}^{\mathrm{h}}$ he subfield of thermal photonics is attracting a lot of attention due to the rapid technological progress in nanophotonics and the promise that these developments can be utilized for thermal radiation management. ${ }^{1-4}$ In this respect, researchers are challenged to develop novel protocols that tackle a variety of bottlenecks imposed by fundamental limitations dictating thermal radiation. For example, a current research effort aims to bypass the constraints set by Kirchoff's and Planck's laws by capitalizing on the importance of evanescent waves in case of subwavelength photonic circuits. $^{5-11}$ In parallel, other studies exploit the applicability of recent proposals for radiation control to daytime passive radiative cooling, ${ }^{12-16}$ radiative cooling of solar cells, ${ }^{17,18}$ energy harvesting, ${ }^{19-24}$ thermal camouflage, ${ }^{25,26}$ and so on.

Along these lines, an important milestone for thermal radiation management is the implementation of photonic protocols that allow for a nonreciprocal heat transfer. The majority of these studies rely on magneto-optical effects ${ }^{27-29}$ or nonlinearities. ${ }^{30-33}$ Only recently, photonic designs that enforce directional radiation transfer via temporal modulations are being explored. ${ }^{34-38}$

In this paper we consider an adiabatic thermal radiation pump that consists of a silicon-based square resonator exchanging, in the near-field, radiation with two thermal reservoirs that are at the same temperature. Two circular domains of the resonator are adiabatically driven by an out-ofphase periodic modulation. We show that, when the cyclic modulation is engineered in a way that its center, in the parameter space of the system, is in the proximity of a resonance degeneracy, the pumped current acquires a maximum value. It is interesting to point out that, on some occasions, resonance degeneracies have been associated with an exceptional point degeneracy of the spectrum of a (nonHermitian) effective Hamiltonian describing the system connected to the reservoirs. ${ }^{34}$ Further design of the pumping cycle for our photonic pump is done following the predictions of a Floquet scattering theory, which, in the adiabatic limit, is utilizing an instantaneous scattering matrix (ISM), and it is confirmed via direct time-domain simulations.

The structure of the paper is as follows. In the next section we present the Floquet scattering formalism and analyze in detail the specific case of adiabatic pumping. Furthermore, utilizing an ISM approximation, we identify conditions that will lead to the design of efficient pumping circles. Based on the insights gained from the ISM Floquet formalism, we design an adiabatic thermal radiation pump using a photonic circuit consisting of a periodically modulated microcavity. The direct time-domain calculations of the radiated thermal current confirm nicely the expectations, thus establishing the ISM Floquet formalism as a useful tool for the design of efficient pumping cycles. Our conclusions are presented at the last section of the paper.

Received: June 17, 2021

Published: September 21, 2021 


\section{THEORETICAL CONSIDERATIONS}

Floquet Scattering Formalism. We consider two thermal reservoirs $\alpha=1$ and 2 at temperature $T_{\alpha}$ that exchange thermal energy via a periodically modulated photonic circuit. The reservoirs emit radiation with amplitudes $\theta_{\alpha}^{+}(\omega)$ that satisfy the condition

$$
\left\langle\left[\theta_{\alpha}^{+}(\omega)\right]^{*} \theta_{\beta}^{+}\left(\omega^{\prime}\right)\right\rangle=\frac{1}{2 \pi} \tilde{\Theta}_{\alpha}(\omega) \delta_{\alpha, \beta} \delta\left(\omega-\omega^{\prime}\right)
$$

where $\langle\cdot\rangle$ indicates a thermal ensemble average, and \langle|$\left.\left.\theta_{\alpha}^{+}(\omega)\right]\left.\right|^{2}\right\rangle$ describes the mean number of $\omega$ photons injected from the $\alpha$-th thermal reservoir to the circuit. The mean photon number is $\tilde{\Theta}_{\alpha}(\omega)=\Phi_{\alpha}(\omega) \cdot \Theta_{\alpha}(\omega)$, where $\Theta_{\alpha}(\omega)=$ $\left(e^{\hbar \omega / k_{\mathrm{B}} T_{\alpha}}-1\right)^{-1}$ and $\Phi_{\alpha}(\omega)$ is a spectral filter function. The latter can be used to control the spectral emissivity of the thermal reservoirs and can be achieved via the deposition of photonic crystals that support band-gaps or their coupling to the photonic circuit via a waveguide or a cavity with cutoff frequencies, and so on. ${ }^{1-3}$

We further assume that the circuit is periodically driven with a set of time-varying parameters $\vec{x}\left(t+\frac{\Omega}{2 \pi}\right)=\vec{x}(t)$ $=\left[x_{1}(t), \ldots, x_{N}(t)\right]^{T}$ that modulate, for instance, the refractive index of its constituent materials. When radiation at frequency $\omega$ enters into the driven circuit, it can be scattered to other sidebands with frequencies $\omega_{m}=\omega+m \Omega, m \in Z$. The incoming/outgoing radiations with amplitudes $\theta_{\alpha}^{ \pm}(\omega)$ from/ toward the reservoir $\alpha$ are related via the Floquet scattering matrix $S^{\mathrm{F}}$ as follows: ${ }^{39-41}$

$$
\theta_{\alpha}^{-}(\omega)=\sum_{\beta, m} \mathbf{s}_{\alpha, \beta}^{\mathrm{F}}\left(\omega, \omega_{m}\right) \theta_{\beta}^{+}\left(\omega_{m}\right)
$$

where the matrix element $\mathbf{S}_{\alpha, \beta}^{\mathrm{F}}\left(\omega_{m,}, \omega_{m}\right)$ describes the transmission/reflection amplitude of the electromagnetic field entering the reservoir $\alpha$ with frequency $\omega_{m^{\prime}}$, provided that it was emitted from reservoir $\beta$ with frequency $\omega_{m}$. We furthermore assume that the number of photons is conserved during the Floquet scattering process, ${ }^{39}$ leading to a unitary Floquet scattering matrix, that is, $\left(\mathbf{S}^{\mathrm{F}}\right)^{\dagger} \mathbf{S}^{\mathrm{F}}=\mathbf{S}^{\mathrm{F}}\left(\mathbf{S}^{\mathrm{F}}\right)^{\dagger}=I$.

We are now ready to evaluate the average photon current toward the reservoir $\alpha$. The latter takes the following form:

$$
\bar{I}_{\alpha}^{\mathrm{Ph}}=\frac{\Omega}{2 \pi} \int_{0}^{2 \pi / \Omega} \mathrm{d} t\left[\left\langle\left|\theta^{-}(t)\right|^{2}\right\rangle-\left\langle\left|\theta^{+}(t)\right|^{2}\right\rangle\right]
$$

where $\theta_{\alpha}^{ \pm}(t)=\int_{0}^{\infty} \theta_{\alpha}^{ \pm}(\omega) e^{-i \omega t} \mathrm{~d} \omega$ is the complex field amplitude. Specifically, using eqs 1 and 2, we obtain

$$
\begin{aligned}
& \bar{I}_{\alpha}^{\mathrm{Ph}}=\int \frac{\mathrm{d} \omega}{2 \pi} N_{\alpha}(\omega), \\
& N_{\alpha}(\omega)=-\tilde{\Theta}_{\alpha}(\omega)+\sum_{\beta, m}\left|\mathbf{S}_{\alpha, \beta}^{F}\left(\omega, \omega_{m}\right)\right|^{2} \tilde{\Theta}_{\beta}\left(\omega_{m}\right)
\end{aligned}
$$

where $N_{\alpha}(\omega)$ is the photon current density entering reservoir $\alpha$.

Similarly, we can evaluate the corresponding thermal radiation energy. The associated average energy current $\bar{I}_{\alpha}$ toward the thermal reservoir $\alpha$ reads:

$$
\begin{aligned}
& \bar{I}_{\alpha}=\int \frac{\mathrm{d} \omega}{2 \pi} \hbar \omega N_{\alpha}(\omega) \\
& =\sum_{\beta, m} \int \frac{\mathrm{d} \omega}{2 \pi} \hbar \omega_{m}\left|\mathbf{S}_{\alpha, \beta}^{F}\left(\omega_{m}, \omega\right)\right|^{2}\left[\tilde{\Theta}_{\beta}(\omega)-\tilde{\Theta}_{\alpha}\left(\omega_{m}\right)\right]
\end{aligned}
$$

where for the second equality we have employed the unitarity of the Floquet scattering matrix $\mathbf{S}^{\mathrm{F}}$. Equations 4 and 5 extend the Landauer-like formalism developed in the framework of mesoscopic quantum electronics ${ }^{39}$ to the case of photons and radiative energy currents through time-modulated circuits. See the detailed derivation of eqs 4 and 5 in the Supporting Information.

Adiabatic Limit of Thermal Radiation. We can do a further analytical progress with eq 5 in the limit of adiabatic modulations, that is, $\Omega \rightarrow 0$, and small temperature gradients. In this case, the Floquet scattering matrix $S^{\mathrm{F}}$ can be expressed in terms of the instantaneous scattering matrix $S^{t}(\omega)$ as $\mathbf{S}^{\mathrm{F}}\left(\omega_{m}, \omega\right)=\mathbf{S}^{\mathrm{F}}\left(\omega, \omega_{-m}\right)=\frac{\Omega}{2 \pi} \int_{0}^{2 \pi / \Omega} \mathrm{d} t S^{t}(\omega) e^{i m \Omega t}$ (see Supporting Information). Substitution of this expression in eq 5 allows us to separate the total energy current, eq 5, into three distinct contributions:

$$
\bar{I}_{\alpha} \approx \bar{I}_{\alpha, b}+\bar{I}_{\alpha, p}+\bar{I}_{\alpha, d}
$$

The first contribution, $\bar{I}_{\alpha, b}$, is due to the temperature gradient between the reservoirs and does not depend on the modulation frequency $\Omega$,

$$
\bar{I}_{\alpha, b}=\int \frac{\mathrm{d} \omega}{2 \pi} \overline{\mathcal{T}}(\omega) \hbar \omega\left[\tilde{\Theta}_{\alpha^{\prime}}(\omega)-\tilde{\Theta}_{\alpha}(\omega)\right]
$$

Here, $\overline{\mathcal{T}}=\frac{\Omega}{2 \pi} \int \mathrm{d} t\left|S_{2,1}^{t}(\omega)\right|^{2}$ is the average transmittance of the frozen system over one modulation cycle. Notice that it is the same for left and right emitted radiation since the reciprocity of the frozen (undriven) system enforces the relation $\left|S_{2,1}^{t}(\omega)\right|^{2}=\left|S_{1,2}^{t}(\omega)\right|^{2}$.

The second contribution $\bar{I}_{\alpha, p}$ is the thermal radiative pumped energy flux, which originates from the modulation of the scatterer and is given as

$$
\bar{I}_{\alpha, p} \approx \frac{\Omega}{2 \pi} \int \frac{\mathrm{d} \omega}{2 \pi} \hbar\left\{\omega \tilde{\Theta}_{0}(\omega) \frac{\partial P_{\alpha}}{\partial \omega}+P_{\alpha}(\omega) \tilde{\Theta}_{0}(\omega)\right\}
$$

where $\tilde{\Theta}_{0}(\omega)=\left.\tilde{\Theta}_{\alpha}(\omega)\right|_{T_{\alpha}=T_{0}}$ is associated with a mean temperature $T_{0}=\left(T_{1}+T_{2}\right) / 2$, and $P_{\alpha}(\omega)=i \int \mathrm{d} t$ $\left(\frac{\mathrm{d} S^{t}}{\mathrm{~d} t}\left(S^{t}\right)^{\dagger}\right)_{\alpha, \alpha}$. In the classical limit $\tilde{\Theta}_{0}(\omega) \approx k_{\mathrm{B}} T_{0} /(\hbar \omega)$, the integration of the first term of eq 8 approaches zero. We note that $\bar{I}_{\alpha, p}$ can be different than zero, even in the absence of a temperature gradient. In addition, the direction of the energy current can be easily controlled by the orientation of the modulation loop in the parameter space.

Finally, the third term, $\bar{I}_{\alpha, d}$, in eq 6 is the analogue of a dissipation term found in adiabatic quantum pumps in condensed matter physics ${ }^{39,45-47}$ and reads 


$$
\begin{aligned}
\bar{I}_{\alpha, d}= & \frac{\Omega^{2}}{2 \pi} \int \frac{\mathrm{d} \omega}{2 \pi} \hbar\left[\oint_{0}^{2 \pi} \mathrm{d} x\left(\frac{\partial S^{x}}{\partial x} \frac{\partial\left(S^{x}\right)^{\dagger}}{\partial x}\right)_{\alpha, \alpha}\right] \\
& \times\left[-\frac{\partial \tilde{\Theta}_{\alpha}(\omega)}{\partial \omega}-\frac{\omega}{2} \frac{\partial^{2} \tilde{\Theta}_{\alpha}(\omega)}{\partial \omega^{2}}\right]
\end{aligned}
$$

where $x=\Omega t$. In contrast to the pumped current, $\bar{I}_{\alpha, d}$ is proportional to $O\left(\Omega^{2}\right)$, and its direction does not depend on the orientation of the modulation loop, that is, the substituting of $\Omega \rightarrow-\Omega$ does not reverse the direction of this current contribution. Moreover, in the adiabatic limit, the dissipative current is negligible as compared to the pumped current, which scales as $O(\Omega)$. Obviously, the smaller the value of $\bar{I}_{\alpha, d}$, the better is the performance of an adiabatic thermal pump.

Adiabatic Pumping Current. Of particular interest is the analysis of the adiabatic energy flux between two reservoirs at the same temperature. In such a scenario, known as adiabatic thermal radiation pumping, ${ }^{34}$ the thermal radiative flux is dominated by the pumping current contribution. This can be evaluated using eq 8 and employing the slow modulation $\Omega \rightarrow$ 0 limit. We get

$$
\begin{aligned}
& \bar{I}_{\alpha} \approx \frac{\Omega}{2 \pi} \int \frac{\mathrm{d} \omega}{2 \pi} \hbar \omega \tilde{\Theta}_{0}(\omega) \mathcal{A} Q_{\alpha}(\omega), \\
& Q_{\alpha}(\omega)=\lim _{\mathcal{A} \rightarrow 0+} \frac{1}{\mathcal{A}}\left\{\frac{1}{\omega} \frac{\partial\left[\omega P_{\alpha}(\omega)\right]}{\partial \omega}\right\}
\end{aligned}
$$

where $Q_{\alpha}(\omega)$ represents the radiative energy density per pumping area, and we have also assumed that the modulation cycle encloses a small area in the parameter space, that is, $\mathcal{A}=\oint \Pi_{\nu=1}^{N} \mathrm{~d} X_{\nu} \rightarrow 0$. In the derivation of eq 10 , we have also omitted the dissipative term $I_{\alpha, d}$ since it is proportional to $O\left(\Omega^{2}\right)$.

It is interesting to note that $P_{\alpha}$ (and thus $Q_{\alpha}$ ) can be given conveniently in terms of the instantaneous scattering matrix $S^{t}$. To better understand this, we parametrize $S^{t}$ as

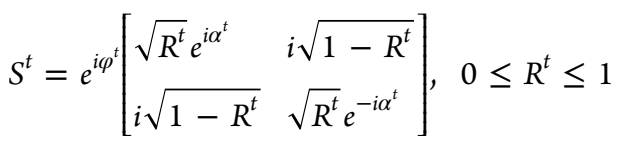

where $\varphi^{t}$ is the transmission phase, $R^{t}$ is the reflectance, and $\alpha^{t}$ is the phase of the reflection coefficient. With this parametrization, we can show that

$$
P_{1}=-P_{2}=\int_{0}^{2 \pi / \Omega} \mathrm{d} t R^{t} \frac{\mathrm{d} \alpha^{t}}{\mathrm{~d} t}
$$

which highlights the geometric character of the pumping operation. $^{42,43}$ Equation 12 also constitutes a dramatic computational simplification for the evaluation of $\bar{I}_{\alpha}$ (compare with eqs 13 and 14 below).

On the other hand, the radiative (time-averaged) pumped thermal energy flux $\bar{I}_{\alpha}$ during one pumping cycle can be evaluated directly from direct time domain experiments using the following expression ${ }^{34}$

$$
\bar{I}_{\alpha} \equiv \frac{\Omega}{2 \pi} \int_{0}^{2 \pi / \Omega} \mathrm{d} t \int \frac{\mathrm{d} \omega}{2 \pi} \mathcal{I}_{x_{0}}(t, \omega) \hbar \omega \tilde{\Theta}_{0}(\omega)
$$

where $\mathcal{I}_{x_{0}}$ is the normalized time-dependent directional net energy current evaluated at some observation position $x_{0}$ between the system and the reservoir. Comparison between eqs 10 and 13 allows us to identify the radiative energy density (i.e., per area in the parameter space) $Q(\omega)$ to be equal to

$$
Q(\omega) \equiv \lim _{\mathcal{A} \rightarrow 0} \frac{1}{\mathcal{A}} \int_{0}^{2 \pi / \Omega} \mathrm{d} t \mathcal{I}_{x_{0}}(t, \omega)
$$

Clearly, the evaluation of the pumping current via eq 14 requires the knowledge of $\mathcal{I}_{x_{0}}(t, \omega)$ during the whole period $t$ $\in[0,2 \pi / \Omega)$ and for all emission frequencies $\omega$. This is a heavy computational effort in case of adiabatic drivings. For its calculation, we will consider uncorrelated thermal emissions at frequency $\omega$ of unit incident flux from the two reservoirs. In our realistic example below with an adiabatically modulated resonator, we shall utilize both expressions and confirm their equivalence.

Design Rules for Efficient Pumping Circles. A careful analysis of the integrand in eq 10 allows us to derive a number of design rules for the engineering of efficient adiabatic thermal radiation pumps. First observation stems from the fact that an appropriate design of the spectral filtering function $\Phi(\omega)$ can lead to an enhancement/suppression of specific frequency components of $Q(\omega)$, thus, controlling the integral in eq 10 and therefore the value of $\bar{I}_{\alpha}$. At the same time, we observe that even in the absence of spectral filtering (i.e., $\Phi_{\alpha}(\omega)=1$ ), the smooth positive function $\omega \tilde{\Theta}_{0}(\omega)$ introduces a frequency weight that suppresses the high-frequency components of $Q_{\alpha}$, while enhancing its corresponding low-frequency ones. Therefore, knowledge of $Q(\omega)$ through the ISM approximation may guide the design of efficient pumping circles.

Another observation is associated with the dependence of $P(\omega)$ from the scattering parameters, see eq 12. This dependence allows us to conclude that the main contribution to $\overline{\mathcal{I}}$ originates from a frequency range around transmission resonances, where $P(\omega)$ becomes significant due to the rapid changes of the instantaneous reflection phase $\alpha^{t}$ and reflectance $R^{t}$. It is natural to assume that when a pair of nearby resonances approach one-another, they can further enchance the sensitivity of $\alpha^{t}$ and $R^{t}$ on the parameters of the pump, thus increasing the pumping-induced thermal energy flux density $\overline{\mathcal{I}}$. These observations will be allowing us to understand and engineer the radiation current of a realistic photonic pump as we will show in the next section.

\section{MICROCAVITY IMPLEMENTATION}

We are now ready to evaluate the thermal energy flux for a realistic photonic adiabatic pump consisting of a square optical microcavity, see Figure 1. The cavity has a linear length $a=50$ $\mu \mathrm{m}$, and it is formed by a dielectric material (silicon) with a relative permittivity $\varepsilon=11.6$ (silicon). We assume that the cavity is surrounded with perfect electric conductors (PECs). The cavity is brought in the proximity of two reservoirs with the same temperature $T=300 \mathrm{~K}$.

We adiabatically drive the cavity by modulating the permittivities $\varepsilon_{1(2)}(t)$ within two embedded circular spots, while the permeability $\mu_{0}$ remains constant, see Figure 1 . In our simulations below, we will consider the specific modulation protocol $\varepsilon_{1(2)}=\varepsilon\left(U+\Delta \varepsilon_{1(2)}\right)$, where $\left(\Delta \varepsilon_{1}, \Delta \varepsilon_{2}\right)=$ $\frac{\Delta}{\varepsilon}(-\sin \Omega t, \cos \Omega t)$ and the offset $U$ shifts the driving circle along the line $\varepsilon_{1}=\varepsilon_{2}=\varepsilon U$ in the parameter space $\left(\Delta \varepsilon_{1}, \Delta \varepsilon_{2}\right)$. This type of time-modulation of the dielectric constant of silicon could be achieved via carrier injection with frequency 


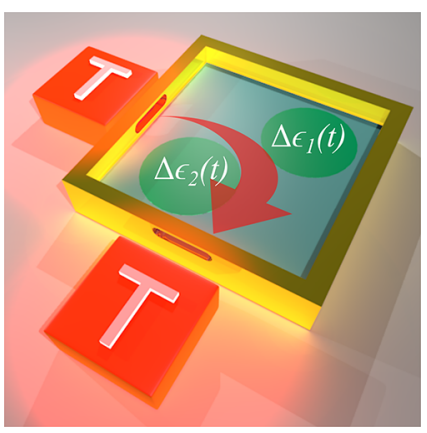

Figure 1. Microcavity for adiabatic thermal radiation pumping. Scheme of a two-dimensional microcavity coupled to two thermal baths at the same temperature $T$. The uniform permittivity $\varepsilon$ filling the cavity undergoes perturbations $\Delta \varepsilon_{1}$ and $\Delta \varepsilon_{2}$ that, when periodically modulated in time, produce a nonzero current between reservoirs.

up to several GHz. ${ }^{44}$ Our goal is to design efficient pumping circles using the ISM Floquet formalism and confirm their efficiency by evaluating the pumped current using direct timedomain simulations.

Eigenmode Analysis. Before analyzing the pumped energy current, we will first describe the spectral properties of the microcavity and the parametric change of the eigenmodes with respect to the permittivity variations $\varepsilon(x, y)$ $=\varepsilon[1+\Delta \varepsilon(x, y)]$. To this end, we first consider the uniform square cavity of size $a$ by setting $\Delta \varepsilon(x, y)=0$. Due to its geometric symmetry, the microcavity presents spectral degeneracies which we will be using for the design of optimal pumping circles.

The spectrum can be evaluated explicitly by solving directly the frequency-domain Maxwell's equations

$$
\nabla \times \vec{E}=-i \omega \mu_{0} \vec{H} ; \quad \nabla \times \vec{H}=-i \omega \varepsilon_{0} \vec{E}
$$

We assume transverse electric (TE) modes of the form $\vec{E}=\vec{E}_{t}(x, y), \vec{H}=\vec{H}_{t}(x, y)+H_{z}(x, y) \hat{z}$, where the vectors $\vec{E}_{t}(x, y)$ and $\vec{H}_{t}(x, y)$ with the subscript $t$ stand for transverse field components in the $x-y$ plane. From eq 15, we have $\vec{H}_{t}=0$ and $\vec{E}_{t}=(i \omega \varepsilon)^{-1} \nabla_{t} H_{z} \times \hat{z}$, leading to the $2 \mathrm{D}$ Helmholtz equation

$$
\nabla_{t}^{2} H_{z}(x, y)+\omega^{2} \varepsilon \mu_{0} H_{z}(x, y)=0
$$

that when supplemented with the PEC boundary condition, that is, $\frac{\partial H_{z}}{\partial n}=0$ yields

$$
H_{z}^{m n}=H_{m n} \cos \left(k_{x} x\right) \cos \left(k_{y} y\right)
$$

where $k_{x}=m \pi / a, k_{y}=n \pi / a$, with $n, m=0,1,2, \ldots$ The corresponding frequency spectrum is

$$
\omega_{m n}=\frac{\pi}{a \sqrt{\varepsilon \mu_{0}}} \sqrt{m^{2}+n^{2}} \quad n, m=0,1,2, \ldots
$$

where $\varepsilon=\varepsilon_{0} \varepsilon_{r}$ is the permittivity and $a$ is the linear size of the square cavity.

We focus on the two degenerate TE modes with the lowest frequencies $\omega_{d} /(2 \pi)=\omega_{10} /(2 \pi)=\omega_{01} /(2 \pi)=\left(2 a \sqrt{\varepsilon \mu_{0}}\right)^{-1} \approx 0.8802$ $\mathrm{THz}$, and the corresponding electric field profiles

$$
\begin{aligned}
& \vec{E}^{01}=E_{x}^{01} \hat{x} ; \quad E_{x}^{01}=i \sqrt{\frac{\mu_{0}}{\varepsilon}} \sqrt{\frac{2}{a}} \sin \left(\frac{\pi}{a} y\right) \\
& \vec{E}^{10}=E_{y}^{10} \hat{y} ; \quad E_{y}^{01}=-i \sqrt{\frac{\mu_{0}}{\varepsilon}} \sqrt{\frac{2}{a}} \sin \left(\frac{\pi}{a} x\right)
\end{aligned}
$$

The modification of the permittivities $\varepsilon_{1(2)}$ lifts the degeneracy. Accordingly, the mode profiles will be modified and their electric fields will satisfy the following relation

$$
\nabla \times \nabla \times \vec{E}=(\omega / c)^{2}(1+\Delta \varepsilon) \vec{E}
$$

We approximate the solution near $\omega_{d}$ via the linear combination $\vec{E} \approx \varphi_{1} \vec{E}^{01}+\varphi_{2} \vec{E}^{10}$ and obtain the following relation using eq 20

$$
\left(\frac{\omega_{d}^{2}-\omega^{2}}{c^{2}}\right)\left(\varphi_{1} \vec{E}^{01}+\varphi_{2} \vec{E}^{10}\right)=\left(\frac{\omega}{c}\right)^{2} \Delta \varepsilon\left(\varphi_{1} \vec{E}^{01}+\varphi_{2} \vec{E}^{10}\right)
$$

which, after taking the dot product with the vectors $\vec{E}^{01^{*}}$ and $\vec{E}^{10^{*}}$ and the subsequent integral over space, yields

$$
\left(\frac{\omega_{d}^{2}-\omega^{2}}{\omega^{2}}\right)\left(\begin{array}{ll}
D^{0} & 0 \\
0 & D^{0}
\end{array}\right)\left(\begin{array}{l}
\varphi_{1} \\
\varphi_{2}
\end{array}\right)=\left(\begin{array}{ll}
N^{0} & N^{01} \\
N^{10} & N^{1}
\end{array}\right)\left(\begin{array}{l}
\varphi_{1} \\
\varphi_{2}
\end{array}\right)
$$

where $D^{0}=\iint\left(\vec{E}^{01}\right)^{*} \cdot \vec{E}^{01} \mathrm{~d} x \mathrm{~d} y=\iint\left(\vec{E}^{10}\right)^{*} \cdot \vec{E}^{10} \mathrm{~d} x \mathrm{~d} y, N^{0}=$ $\iint\left|\vec{E}^{01}\right|^{2} \Delta \varepsilon \mathrm{d} x \mathrm{~d} y, N^{1}=\iint\left|\vec{E}^{10}\right|^{2} \Delta \varepsilon \mathrm{d} x \mathrm{~d} y$, and $N^{10}=N^{01 *}$ $=\int \vec{E}^{10^{*}} \cdot \vec{E}^{01} \Delta \varepsilon \mathrm{d} x \mathrm{~d} y$.

The existence of the nonzero solution of eq 22 allows us to find the frequencies of the perturbed modes:

$$
\frac{\Delta \omega}{\omega_{d}} \approx-\frac{N^{0}+N^{1} \pm \sqrt{\left(N^{0}-N^{1}\right)^{2}+4 N^{01} N^{10}}}{4 D^{0}}
$$

where $\Delta \omega=\omega-\omega_{d}$. It is important to notice that $\vec{E}^{01}$ is perpendicular to $\vec{E}^{10}$, which results in $N^{10}=\left(N^{01}\right)^{*}=0$. Consequently, the splitting of the eigenfrequencies $\Delta \omega$ would vanish when $N^{0}=N^{1}$, producing a degenerate line in the parameter space.

The condition $N^{0}=N^{1}$ for degeneracy is not related to the symmetry of the system. For example, consider the case of a material perturbation on two separate domains with $\Delta \varepsilon(x, y)=$ $\Delta \varepsilon_{1}(x, y)+\Delta \varepsilon_{2}(x, y)$. In this case, we have

$$
\begin{aligned}
N^{0}-N^{1}= & \frac{\mu_{0}}{\varepsilon} \frac{2}{a} \iint \mathrm{d} x \mathrm{~d} y\left[\sin ^{2}\left(\frac{\pi}{a} x\right)+\sin ^{2}\left(\frac{\pi}{a} y\right)\right] \\
& \times\left[\Delta \varepsilon_{1}(x, y)+\Delta \varepsilon_{2}(x, y)\right]
\end{aligned}
$$

Therefore, for a given $\Delta \varepsilon_{1}(x, y)$, we can easily find a $\Delta \varepsilon_{2}(x, y)$ such that $N^{0}-N^{1}=0$. In such a case, the degeneracy will survive even in the presence of these specific permittivity perturbations, which evidently destroy the geometric symmetry of the cavity. Of course, the above claim is correct up to first order perturbation. It is therefore imperative to analyze numerically their existence and even question it in the case that the cavity is coupled to the reservoirs. This latter situation needs additional care since the coupling to the reservoirs introduces an additional perturbation that might destroy the degeneracy of the associated resonant modes. 
To confirm the conclusions of the perturbation argument, we analyze in detail the transmission spectrum of the cavity in a frequency range containing the two lowest resonances, see Figure 2. Due to the coupling to the reservoirs, the resonant

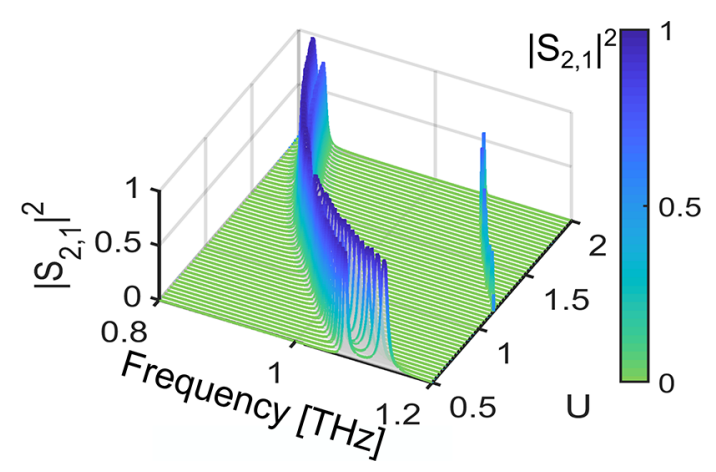

Figure 2. Transmittance spectrum $\left|S_{2,1}\right|^{2}$ of the cavity (in absence of driving) as a function of frequency $\omega /(2 \pi)$ and $U$. The peaks of the transmittance show a resonance degeneracy around $U=1.05$.

frequencies are detuned with respect to the eigenfrequencies of the isolated resonator, leading to a destruction of the degenerate line. The position of the resonant modes as a function of the offset parameter $U$ is indicated in Figure 2 with solid and dashed black lines. We are able to identify a degenerate point where the resonances coalesce as a function of the shift parameter $U$. The degeneracy, occurring at $\omega_{\mathrm{d}} /$ $(2 \pi) \approx 0.95 \mathrm{THz}$ for an offset value $U_{\mathrm{d}}=1.05$, will be the starting point for the design of an efficient pumping circle.

Pumping Cycles and Optimal Thermal Radiation Currents. We assume that the two thermal reservoirs that are attached to the microcavity are at fixed temperature $T=$ $300 \mathrm{~K}$. We model these two reservoirs as two identical lumped ports with characteristic impedance $Z_{0}=50 \mathrm{Ohms}$ and length $\frac{1}{20} a$ (for the specific arrangement, see Figure 1 and Supporting Information). The ports carry time-dependent voltage excitations originating from synthesized noise sources $V_{\alpha}$, which satisfy the correlation relation $\left\langle V_{\alpha}(\omega) V_{\alpha}^{*}\left(\omega^{\prime}\right)\right\rangle=$ $\frac{2 Z_{0}}{\pi} \hbar \omega \Phi(\omega) \delta\left(\omega-\omega^{\prime}\right) \chi_{\alpha, \alpha}$, with $\Theta_{\mathrm{C}}(\omega)=k_{\mathrm{B}} T /(\hbar \omega)$.

In order to isolate the frequency domain where the resonance degeneracy $\omega_{\mathrm{d}} /(2 \pi) \approx 0.95 \mathrm{THz}$ occurs, we have filtered the emission of the baths using a spectral filtering function $\Phi(\omega)=\theta_{\mathrm{H}}\left(\omega-\omega_{\min }\right)-\theta_{\mathrm{H}}\left(\omega-\omega_{\max }\right)$, where $\theta_{\mathrm{H}}(\omega)$ is the Heaviside step function, $\omega_{\min } /(2 \pi)=0.88 \mathrm{THz}$, and $\omega_{\max } /(2 \pi) \leq 1.06 \mathrm{THz}$ was chosen in a way that maximizes the radiative current.

Next, we calculate the radiative energy density $Q(\omega)$ versus frequency. Although the evaluation of $Q(\omega)$ is a demanding computational task in the case of a direct implementation of eq 14 , the simplicity of the ISM approach, eq 10, allows us to perform this task easily for a large number of $U$ values. The results of our calculation are shown in Figure 3. We find that $Q(\omega)$ is significantly enhanced at frequencies $\omega$, which are at the vicinity of the resonance frequencies (indicated with solid and dashed black lines in Figure 3). Importantly, this enhancement turns out to be more pronounced in the neighborhood of the resonance degeneracy around $U_{\mathrm{d}} \approx$ 1.05. In this case, $Q(\omega)$ becomes symmetric around $\omega_{\mathrm{d}}$ where it acquires its maximum magnitude. For slightly different $U$ values, for example, $U=U_{\mathrm{d}} \pm 0.05$, the $Q(\omega ; U)$ becomes

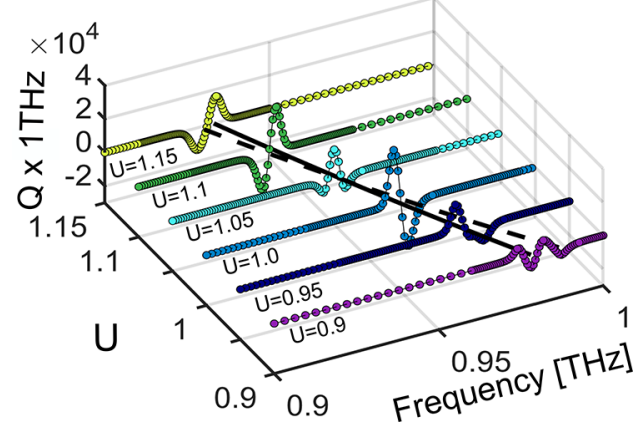

Figure 3. Radiative pumped energy density $Q(\omega)$ versus frequency $\omega /(2 \pi)$ for different values of $U$. The driving protocol is given by the parametric cycle $\varepsilon_{1(2)}=\varepsilon\left(U+\Delta \varepsilon_{1(2)}\right)$, where $\left(\Delta \varepsilon_{1}, \Delta \varepsilon_{2}\right)=$ $\frac{\Delta}{\varepsilon}(-\sin x, \cos x)$ for $x \in[0,2 \pi)$. The resonant frequencies, fitted from the peaks of $\left|S_{2,1}\right|^{2}$, are shown with black solid and dashed lines. The highest values of $Q(\omega)$ are obtained at $U=1$ and $U=1.1$, which are in the neighborhood of the resonance degeneracy. For the calculation of $Q$ we used a parametric area $\mathcal{A}=\pi(\Delta / \varepsilon)^{2}, \Delta=0.2,{ }^{48}$ $\varepsilon=11.6$, and frequency range $\left[\omega_{\min }, \omega_{\max }\right) /(2 \pi)=[0.88,1.06) \mathrm{THz}$.

antisymmetric (see Figure 3), developing two resonant peaks symmetrically placed around a particular frequency $\omega_{\mathcal{c}}$, where $Q\left(\omega_{c} ; U\right)=0$.

The integration of $Q(\omega)$ with the weight function $\hbar \omega \Theta_{\alpha}(\omega)$ (we assume $\Phi(\omega)=1$ ) leads to a slightly enhanced contribution of the smaller frequencies in the radiative pumped current $\bar{I}_{\alpha}$, eq 10 . However, the antisymmetric shape of $Q(\omega)$ affects the calculation for $\bar{I}_{\alpha}$ such that it turns out to be (almost) zero. Of course, one can further the enhancement of the magnitude of the radiative current by appropriately engineering the spectral filtering function that applies at the reservoirs. In Figure 4, we report the thermal radiation current $\bar{I}_{\alpha}$ versus the control parameter $U$ for an example case of a spectral filter such that $\omega_{\max }=\omega_{\mathrm{d}}+a \cdot\left(U-U_{\mathrm{d}}\right)$, with $a \approx$ $-1.84 \times 10^{11}$, that fits the resonances around $U \approx U_{\mathrm{d}}$. Such

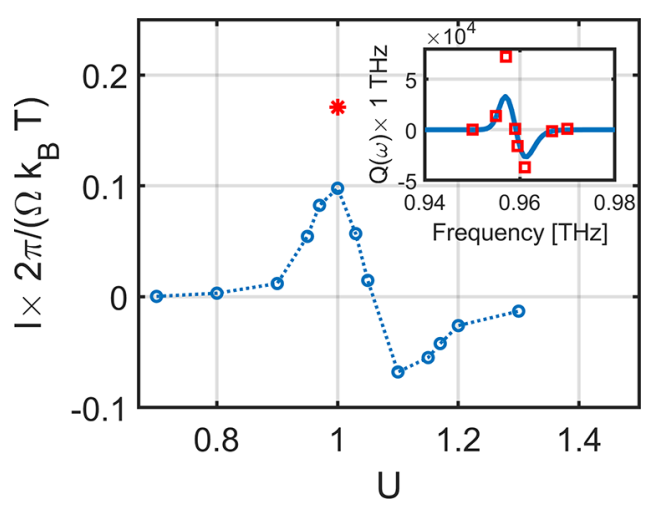

Figure 4. Total (averaged) pumped radiative energy current $\bar{I}$ vs $U$ from the integration in frequency of $Q(\omega)$ according to eq 10, blue circles joined through a dotted line. The red asterisk indicates the associated radiative energy current from the time domain simulations. (Inset) Average pumped radiative energy density $Q(\omega)$ vs frequency $\omega /(2 \pi)$ for $U=1$. The solid blue line is obtained from the scattering parameters through eq 10, and the red square dots are the results of the time domain simulation. For the latter we used a modulation frequency $\Omega=0.0125 \omega_{0}$, where $\omega_{0} \approx 2 \pi \times 1 \mathrm{THz}$. We set the total time to $0.5 \mathrm{~ns}$, which contains at least five modulation cycles, ensuring a fairly reasonable steady state. 
filtering enforces a complete suppression of the large $\omega$ contributions to the integral of eq 10 , leading to a dramatic increase of the radiation current. Since the radiative energy density is also antisymmetric with respect to the offset parameter $U$ around the degeneracy point $U_{\mathrm{d}}$, the "sign" of the first peak will change as we are moving across $U_{\mathrm{d}}$. As a result, the relative position (with respect to $U_{\mathrm{d}}$ ) of the driving cycle in the parameter space can be used as a reconfigurable "knob" for managing the direction of the pumping flux $\bar{I}$. At the same figure, we also report (red star) the total radiation current using a direct implementation of eqs 13 and 14 for the case of $U=1$. The corresponding $Q(\omega)$ has been evaluated via time-domain simulations using eq 14 and it is shown in the inset of Figure $4 \mathrm{~b}$. For these simulations, the photonic cavity has been illuminated with uncorrelated excitations of unit flux via the ports that has been generated using monochromatic voltage sources. Once the steady state is achieved, we integrated the average net power over one modulation cycle to obtain $Q(\omega)$ by employing eq 14 . The nice agreement between these two methods (see blue line and red squares in the inset) confirms the efficiency of the instantaneous scattering matrix approach.

\section{CONCLUSION}

We have developed a Floquet scattering formalism that allows us to design periodic, time-modulation schemes for thermal radiation management. In the adiabatic limit, we arrived to analytical expressions where the net average radiative energy current can be separated into three contributions identified as bias current, pumped energy current, and dissipation due to the driving. All these components have been conveniently expressed in terms of the instantaneous scattering matrix. The resulting expressions allow for better insight into the problem of radiation management. As an example, we have used them to enhance the radiation current in the case of an adiabatic thermal pump corresponding to the scenario of thermal reservoirs with equal temperature. We validate the analytical results by performing realistic time domain simulations in a photonic microcavity setup. We show that when a pumping cycle is designed to operate in the proximity of a spectral degeneracy, the pumped energy flux is dramatically enhanced. Furthermore, the direction of the radiative current can be reconfigured by displaying the position of the center of the pumping cycle with respect to the degeneracy point in the parameter space. Our results can be used for the study of efficient thermal radiative machines, and they offer a new computational tool that allows us to design efficient driving protocols for radiation management.

\section{ASSOCIATED CONTENT}

\section{SI Supporting Information}

The Supporting Information is available free of charge at https://pubs.acs.org/doi/10.1021/acsphotonics.1c00896.

Relation between Floquet and instantaneous scattering matrices; Detailed calculations of the thermal radiative current and its adiabatic limit; Energy balance in periodically modulated setups; Scattering analysis of the microcavity setup (PDF)

\section{AUTHOR INFORMATION}

\section{Corresponding Author}

Lucas J. Fernández-Alcázar - Wave Transport in Complex Systems Lab, Department of Physics, Wesleyan University, Middletown, Connecticut 06459, United States; Instituto de Modelado e Innovación Tecnológica (CONICET-UNNE) and Facultad de Ciencias Exactas, Naturales y Agrimensura, Universidad Nacional del Nordeste, W3404AAS Corrientes, Argentina; 10 orcid.org/0000-0002-1423-6706;

Email: lfernandez_at_exa.unne.edu.ar

\section{Authors}

Huanan Li - Photonics Initiative, Advanced Science Research Center, CUNY, New York, New York 10031, United States

Mona Nafari - Wave Transport in Complex Systems Lab, Department of Physics, Wesleyan University, Middletown, Connecticut 06459, United States

Tsampikos Kottos - Wave Transport in Complex Systems Lab, Department of Physics, Wesleyan University, Middletown, Connecticut 06459, United States

Complete contact information is available at:

https://pubs.acs.org/10.1021/acsphotonics.1c00896

\section{Notes}

The authors declare no competing financial interest.

\section{ACKNOWLEDGMENTS}

We acknowledge partial support by an ONR Grant No. N00014-16-1-2803, by a NSF Grant No. EFMA-1641109, and by a Grant No. 733698 from Simons Collaboration in MPS. L.J.F.-A. acknowledges partial financial support from CONICET.

\section{REFERENCES}

(1) Fan, S. Thermal photonics and energy applications. Joule 2017, 1 (2), 264-273.

(2) Li, W.; Fan, S. Nanophotonic control of thermal radiation for energy applications. Opt. Express 2018, 26 (12), 15995-16021.

(3) Cuevas, J. C.; García-Vidal, F. J. Radiative Heat Transfer. ACS Photonics 2018, 5, 3896.

(4) Baranov, D. G.; Xiao, Y.; Nechepurenko, I. A.; Krasnok, A.; Alu, A.; Kats, M. A. Nanophotonic engineering of far-field thermal emitters. Nat. Mater. 2019, 18, 920-930.

(5) Zhu, L.; Fan, S. Near-complete violation of detailed balance in thermal radiation. Phys. Rev. B: Condens. Matter Mater. Phys. 2014, 90, 220301.

(6) Hadad, Y.; Soric, J. C.; Alú, A. Breaking temporal symmetries for emission and absorption. Proc. Natl. Acad. Sci. U. S. A. 2016, 113 (13), $3471-3475$.

(7) Greffet, J.-J.; Bouchon, P.; Brucoli, G.; Marquier, F. Light Emission by Nonequilibrium Bodies: Local Kirchhoff Law. Phys. Rev. $X$ 2018, 8 (2), 021008.

(8) Biehs, S. A.; Ben-Abdallah, P. Revisiting super-Planckian thermal emission in the far-field regime. Phys. Rev. B: Condens. Matter Mater. Phys. 2016, 93 (16), 165405.

(9) Maslovski, S. I.; Simovski, C. R.; Tretyakov, S. A. Overcoming blackbody radiation limit in free space: metamaterial superemitter. New J. Phys. 2016, 18, 013034.

(10) Fernández-Hurtado, V.; Fernández-Domínguez, A. I.; Feist, J.; García-Vidal, F. J.; Cuevas, J. C. Super-Planckian far-field radiative heat transfer. Phys. Rev. B: Condens. Matter Mater. Phys. 2018, 97, 045408 .

(11) Fernández-Hurtado, V.; Fernández-Domínguez, A. I.; Feist, J.; García-Vidal, F. J.; Cuevas, J. C. Exploring the limits of Super- 
Planckian far-field radiative heat transfer using 2D materials. ACS Photonics 2018, 5 (8), 3082-3088.

(12) Rephaeli, E.; Raman, A.; Fan, S. Ultrabroadband photonic structures to achieve high-performance daytime radiative cooling. Nano Lett. 2013, 13 (4), 1457-1461.

(13) Raman, A. P.; Anoma, M. A.; Zhu, L.; Rephaeli, E.; Fan, S. Passive radiative cooling below ambient air temperature under direct sunlight. Nature 2014, 515, 540-544.

(14) Gentle, A. R.; Smith, G. B. A subambient open roof surface under the mid-summer. Adv. Sci. 2015, 2 (9), 1500119.

(15) Kou, J.; Jurado, Z.; Chen, Z.; Fan, S.; Minnich, A. J. Daytime radiative cooling using near-black infrared emitters. ACS Photonics 2017, 4, 626-630.

(16) Zhai, Y.; Ma, Y.; David, S. N.; Zhao, D.; Lou, R.; Tan, G.; Yang, R.; Yin, X. Scalable-manufactured randomized glass-polymer hybrid metamaterial for daytime radiative cooling. Science 2017, 355, 10621066.

(17) Zhu, L.; Raman, A.; Wang, K. X.; Anoma, M. A.; Fan, S. Radiative cooling of solar cells. Optica 2014, 1, 32-38.

(18) Li, W.; Shi, Y.; Chen, K.; Zhu, L.; Fan, S. A comprehensive photonic approach for solar cell cooling. ACS Photonics 2017, 4 (4), 774-782.

(19) Green, M. A. Time-Asymmetric Photovoltaics. Nano Lett. 2012, 12 (11), 5985-5988.

(20) Lenert, A.; Bierman, D. M.; Nam, Y.; Chan, W. R.; Celanović, I.; Soljačíc, M.; Wang, E. N. A nanophotonic solar thermophotovoltaic device. Nat. Nanotechnol. 2014, 9, 126-130.

(21) Latella, I.; Pérez-Madrid, A.; Rubi, J. M.; Biehs, S.-A.; BenAbdallah, P. Heat Engine Driven by Photon Tunneling in Many-Body Systems. Phys. Rev. Appl. 2015, 4, 011001.

(22) Zhou, Z.; Sakr, E.; Sun, Y.; Bermel, P. Solar thermophotovoltaics: reshaping the solar spectrum. Nanophotonics 2016, 5 (1), 121.

(23) Bierman, D. M.; Lenert, A.; Chan, W. R.; Bhatia, B.; Celanović, I.; Soljačić, M.; Wang, E. N. Enhanced photovoltaic energy conversion using thermally based spectral shaping. Nat. Energy 2016, 1 (6), 16068.

(24) Fiorino, A.; Zhu, L.; Thompson, T.; Mittapally, R.; Reddy, P.; Meyhofer, E. Nanogap near-field thermophotovoltaics. Nat. Nanotechnol. 2018, 13, 806-811.

(25) Li, Y.; Bai, X.; Yang, T.; Luo, H.; Qiu, C.-W. Structured thermal surface for radiative camouflage. Nat. Commun. 2018, 9 (1), 273.

(26) Kats, M. A. Vanadium dioxide as a natural disordered metamaterial: perfect thermal emission and large broadband negative differential thermal emittance. Phys. Rev. X 2013, 3, 041004.

(27) Ben-Abdallah, P. Photon Thermal Hall Effect. Phys. Rev. Lett. 2016, 116, 084301.

(28) Ott, A.; Messina, R.; Ben-Abdallah, P.; Biehs, S.-A. Radiative thermal diode driven by non-reciprocal surface waves. Appl. Phys. Lett. 2019, 114, 163105 .

(29) Ott, A.; Messina, R.; Ben-Abdallah, P.; Biehs, S.-A. Magnetothermoplasmonics: from theory to applications. J. Photonics Energy 2019, 9 (3), 032711.

(30) Ben-Abdallah, P.; Biehs, S.-A. Phase-change radiative thermal diode. Appl. Phys. Lett. 2013, 103, 191907.

(31) Ito, K.; Nishikawa, K.; Iizuka, H.; Toshiyoshi, H. Experimental investigation of radiative thermal rectifier using vanadium dioxide. Appl. Phys. Lett. 2014, 105, 253503.

(32) Fiorino, A.; Thompson, D.; Zhu, L.; Mittapally, R.; Biehs, S.-A.; Bezencenet, O.; El-Bondry, N.; Bansropun, S.; Ben-Abdallah, P.; Meyhofer, E.; Reddy, P. A Thermal Diode Based on Nanoscale Thermal Radiation. ACS Nano 2018, 12 (6), 5774-5779.

(33) Khandekar, C.; Lin, Z.; Rodriguez, A. W. Thermal radiation from optically driven Kerr $(\chi(3))$ photonic cavities. Appl. Phys. Lett. 2015, 106, 151109 .

(34) Li, H.; Fernández-Alcázar, L. J.; Ellis, F.; Shapiro, B.; Kottos, T. Adiabatic Thermal Radiation Pumps for Thermal Photonics. Phys. Rev. Lett. 2019, 123, 165901.
(35) Buddhiraju, S.; Li, W.; Fan, S. Photonic Refrigeration from Time-Modulated Thermal Emission. Phys. Rev. Lett. 2020, 124, 077402.

(36) Latella, I.; Messina, R.; Rubi, J. M.; Ben-Abdallah, P. Radiative Heat Shuttling. Phys. Rev. Lett. 2018, 121, 023903.

(37) Messina, R.; Ben-Abdallah, P. Many-body near-field radiative heat pumping. Phys. Rev. B: Condens. Matter Mater. Phys. 2020, 101, 165435.

(38) Fernández-Alcázar, L. J.; Kononchuk, R.; Li, H.; Kottos, T. Extreme Nonreciprocal Near-Field Thermal Radiation via Floquet Photonics. Phys. Rev. Lett. 2021, 126, 204101.

(39) Moskalets, M.; Büttiker, M. Floquet scattering theory of quantum pumps. Phys. Rev. B: Condens. Matter Mater. Phys. 2002, 66, 205320.

(40) Li, H.; Kottos, T.; Shapiro, B. Floquet-Network Theory of Nonreciprocal Transport. Phys. Rev. Appl. 2018, 9, 044031.

(41) Li, H.; Shapiro, B.; Kottos, T. Floquet scattering theory based on effective Hamiltonians of driven systems. Phys. Rev. B: Condens. Matter Mater. Phys. 2018, 98, 121101.

(42) Bhandari, B.; Alonso, P. T.; Taddei, F.; von Oppen, F.; Fazio, R.; Arrachea, L. Geometric properties of adiabatic quantum thermal machines. Phys. Rev. B: Condens. Matter Mater. Phys. 2020, 102, 155407.

(43) Trivedi, R.; White, A.; Fan, S.; Vučković, J. Analytic and geometric properties of scattering from periodically modulated quantum-optical systems. Phys. Rev. A: At., Mol., Opt. Phys. 2020, 102, 033707.

(44) Reed, G. T.; Mashanovich, G.; Gardes, F. Y.; Thomson, D. J. Silicon optical modulators. Nat. Photonics 2010, 4, 518-526.

(45) Avron, J. E.; Elgart, A.; Graf, G. M.; Sadun, L. Optimal Quantum Pumps. Phys. Rev. Lett. 2001, 87, 236601.

(46) Avron, J. E.; Elgart, A.; Graf, G. M.; Sadun, L. Transport and Dissipation in Quantum Pumps. J. Stat. Phys. 2004, 116 (1), 425473.

(47) Cohen, D. Quantum pumping and dissipation: From closed to open systems. Phys. Rev. B: Condens. Matter Mater. Phys. 2003, 68, 201303.

(48) In our numerical example below, we have used an index variation of the order of $1 \%$. This is at the high end of achievable index variations and has been used for a numerical efficiency of the time-domain simulations with the goal to compare these results with the predictions of the instantaneous scattering matrix formalism. 